\title{
NOTES SUR LA \\ GRAMMAIRE PHILOSOPHIQUE DES TROPES DE MICHELE PRANDI ${ }^{1}$
}

\author{
Michelange Baudoux ${ }^{2}$
}

La Grammaire philosophique des tropes de M. Prandi peut se comprendre à la fois comme une analyse systématique du champ tropologique (métaphore, métonymie, synecdoque) et, dans une optique moins étriquée, comme un essai philosophique sur la communication langagière. La richesse de l'ouvrage et la profondeur de son écriture proviennent d'ailleurs sans doute de cet incessant va-et-vient entre, d'une part, une visée grammaticale toute empreinte de rigueur descriptive, et, d'autre part, une visée philosophique qui fait rebondir l'observation des faits vers l'horizon en devenir des théories linguistiques contemporaines.

Cependant, dans les lignes qui suivent, nous distinguerons soigneusement ces deux démarches. Ce faisant, nous espérons pouvoir nous affranchir du style toujours dense -et souvent opaque- de l'auteur. Nous entendons ainsi expliciter les lignes de force qui parcourent le texte, de manière à en proposer une propédeutique. Cet exposé sera

1 Michele PRAnd, Grammaire philosophique des tropes, Paris, Éd. de Minuit, 1992, $252 \mathrm{p}$.

2 Assistant au Département de communication de l'Université catholique de Louvain. 
suivi d'une brève lecture critique, visant à mettre l'ouvrage en relation avec la sémantique cognitive, et avec nos propres considérations sur le caractère représentationnel de l'intellection métaphorique.

Afin de faciliter une présentation nécessairement succincte, l'ordre, les titres et la structure des rubriques de notre texte ne correspondent pas à ceux de l'auteur. L'ensemble des graphiques et tableaux ont été imaginés par nous pour les besoins du présent article, de même que certains paradigmes exemplatifs.

Le livre est divisé en deux sections. La première, intitulée $L a$ mise en forme des tropes dans l'énoncé, décrit les structures linguistiques (syntaxe, significations) et ontologiques (représentations) nécessaires et suffisantes à l'existence des tropes.

La seconde section (L'interprétation des tropes dans le discours) explore les interférences entre linguistique et pragmatique des tropes, parcourant la distance qui sépare le contenu sémantique des phrases de leur valeur communicative d'énoncés.

Les deux sections sont articulées autour d'une longue parenthèse, (Interlude: structure sémantique et interprétation discursive des énoncés) qui établit le lien entre les deux sections principales et condense l'essentiel de la visée philosophique de l'ouvrage. Dans cette partie, l'auteur replace la grammaire des tropes dans le cadre plus large d'une éthique herméneutique de la communication, en mettant l'accent sur la responsabilité des participants dans la communication langagière.

\section{Les tropes comme énoncés contradictoires}

L'auteur définit le trope comme "la mise en forme linguistique d'un conflit conceptuel". Il y a trope lorsqu'une connexion linguistique entre deux concepts contredit les frontières conceptuelles, structurales (code) ou ontologiques (représentations). La contradiction qui apparaît dans une métaphore comme La lune rêve provient de la mise en relation de deux concepts réciproquement non pertinents ${ }^{1}$.

Un énoncé tropique est donc un énoncé contradictoire, qui pose une relation entre deux termes dont notre appareil catégoriel présuppose qu'ils sont incompatibles, mais pas opposés. L'énoncé tropique se

1 On retrouve ici la définition de la métaphore par Ricceur (1975) comme prédication impertinente. 
différencie ainsi de l'énoncé directement contradictoire (J'aime et je n'aime pas), qui pose explicitement deux affirmations contradictoires, et de l'oxymore (Une obscure clarté), qui rapproche dans le même syntagme deux termes en opposition polaire dans le même paradigme.

Si le trope relève d'une connexion contradictoire, celle-ci ne peut être observée au seul niveau du mot figuratif (celui qui doit être pris au sens figuré), qui ne constitue que l'une de ses deux extrémités.

A l'autre bout d'une connexion syntaxique, il y a une autre expression, littérale celle-là: rêve n'est figuratif que par connexion contradictoire avec lune. L'étude de tels tropes ne peut se réduire à l'approche atomisante traditionnelle, confinée à l'échelle du mot. Pour les saisir dans leurs nuances et leur vitalité, il est nécessaire d'étudier la phrase: la phrase est en effet le lieu où se nouent les connexions tropiques et où s'articulent les contenus linguistiques complexes. C'est pourquoi Prandi préfère élaborer une grammaire des tropes plutôt qu'une sémiotique'.

A la connexion syntaxique s'oppose la connexion référentielle, qui relie le signifiant tropique, non pas à un autre signifiant, mais à un référent contradictoire. Le signe langagier n'est alors trope que comme énoncé, il n'est pas contradictoire en tant que phrase, en tant que pur signifiant linguistique ${ }^{2}$.

\section{Une grammaire cognitive des tropes}

L'incompatibilité entre concepts interconnectés peut se situer soit au niveau structural, soit au niveau de l'imagination, niveau que Prandi nomme ontologique.

La contradiction formelle (L'homme rugit) a lieu lorsque la connexion transgresse une frontière lexicalisée, contredit la sémantique structurale des paradigmes linguistiques. Dans ce cas, le signifiant tropique (rugit) admet dans le code un substitut non contradictoire (hurle, crie). Prandi parle alors de trope-figure.

1 Se référant à Benveniste, Ricœur (La métaphore vive, Paris, Éd. du Seuil, 1975) entend compléter la classique sémiotique des tropes par une sémantique. Comme Prandi, il cherche à mettre en valeur la complexité structurale et pragmatique de la phrase et de l'énoncé.

2 Le référent incompatible avec le désignateur tropique peut être situationnel (déictique tropique) ou textuel (anaphorique tropique). 
La contradiction conceptuelle (La lune rêve) se présente lorsque la connexion transgresse une "frontière ontologique", contredit la structure implicite de notre représentation imaginaire de l'environnement. Dans ce cas, aucun substitut structural du signifiant tropique n'est disponible en langue. Prandi parle alors de trope non-substitutif.

Telle que Prandi la conçoit, l'ontologie (l'imagination) repose sur une modélisation prélangagière et prélogique du monde extérieur. Elle est de l'ordre de la représentation sociale et culturelle, passant éventuellement au-dessus des frontières entre langues, et s'oppose, en tant que forme de connaissance, au savoir scientifique positiviste et rationnel. C'est l'intervention de l'ontologie dans la construction de nombreux contenus contradictoires qui justifie l'élaboration d'une grammaire philosophique des tropes, que la grammaire purement formelle ne saurait aborder dans toutes leurs dimensions.

Ainsi, le contenu d'un énoncé tropique ne se répartit pas seulement entre signifié et illocution, entre la pureté de la signification et la réalité concrète du sens contextuel, entre le contenu formel et le contenu pragmatique: à l'interface entre ces deux pôles, l'auteur met en relief le rôle du contenu ontologique intuitif, dont les catégories ne se superposent pas exactement aux catégories lexicales.

"Pour pouvoir appliquer les signifiés des énoncés au monde, il faut au préalable rendre les deux ordres de grandeur réciproquement commensurables, et donc traduire contextuellement les contenus conceptuels abstraits et stylisés des énoncés dans le langage des faits empiriques: en d'autres termes, il faut imaginer des modèles d'états du monde empirique compatibles avec les contenus conceptuels et se demander si au moins un de ces modèles est compatible avec les données empiriques relevant de l'état du monde. Une telle transposition de contenus complexes en schèmes perceptifs peut être plus ou moins immédiate en fonction des propriétés systématiques de l'énoncé -comme son caractère plus ou moins abstrait, positif ou négatif, contradictoire ou cohérent- et de la présence d'un contexte plus ou moins favorable, mais représente en tout cas une étape inéliminable sur le chemin de la vérité empirique" (p. 194-195).

C'est là le fonctionnement de base des structures ontologiques: elles mobilisent des modèles mentaux figuratifs analogues aux référents extralinguistiques (le signifié /soleil/ relié au concept de soleil active l'image mentale figurative d'un soleil qui entretient avec le soleil extralinguistique une relation de ressemblance analogique). 
Tout comme la phonologie néglige les traits phonétiques du son sémantiquement non fonctionnels, la sémantique néglige les traits ontologiques des concepts grammaticalement non pertinents. La grammaire pure, si elle décrit la forme du contenu, ne dit pas tout du contenu. La modélisation langagière de notre expérience du monde ne s'explique pas par la seule propriété structurale, oppositive et négative, du lexique. Pour comprendre notre capacité à comprendre les choses à l'aide des mots, il faut prendre en compte les propriétés ontologiques, c'est-à-dire figuratives et positives, des concepts.

Sans faire aucunement référence aux auteurs spécifiquement cognitivistes, Prandi rejoint ici la tendance dominante de la linguistique contemporaine, qui, sous des étiquettes comme "psycholinguistique" "sémantique cognitive"1, "communication analogique", se consacre à l'étude des relations entre signes et modèles mentaux. Formelle et philosophique, la grammaire de Prandi est donc aussi cognitive.

\section{Les restrictions grammaticales à l'existence à tropes}

On peut distinguer les tropes ponctuels, reposant sur une connexion référentielle (cf. supra), des connexions tropiques, où la contradiction, visible au plan de l'expression, repose sur une connexion syntaxique. Le foyer métaphorique nominal est apte à soutenir les deux types de connexion, alors que le verbe, même impersonnel, n'admet d'interprétation métaphorique que sur base d'une contradiction syntaxique.

En position référentielle, c'est-à-dire lorsqu'il est destiné à identifier une instance extralinguistique, le nom peut recevoir tous les tropes: la métonymie (la vengeance à la main pour l'arme à la main), la synecdoque (une voile pour un navire), la métaphore (Cette fille est un rossignol).

En position prédicative, lorsqu'il est destiné à qualifier une instance déjà identifiée, le nom n'est accessible qu'à la métaphore (Selon Prandi, Cette fille est un rossignol est grammatical, *cette voile est un navire ou *ette arme est la vengeance ne le sont pas.)

I Dans ce contexte, le terme de sémantique n'implique pas, comme chez Ricœur, une valorisation de l'échelle de l'énoncé par rapport à l'échelle du mot. 
La vocation première de la métaphore est la qualification. Elle apparaît donc plus facilement en position prédicative et ses emplois déictiques comportent toujours une qualification hautement informative (ce rossignol nous en dit plus sur son référent que cette fille). Inversement, métonymie et synecdoque, qui ont une fonction principalement déictique, n'accèdent à la position prédicative (Cette arme, c'est la vengeance), qu'en vertu d'un affaiblissement de la copule verbale (au sens identitaire du verbe être, l'arme n'est pas autant la vengeance qu'elle n'est, métaphoriquement, le complice de l'assassin).

Les verbes n'admettent d'emplois tropiques que métaphoriques (La bise pleure). Les emplois traditionnellement recensés comme métonymiques (Rougir de ses actes, trembler à cette idée), reposent sur des associations contextuelles qui ne mettent pas en jeu de contenus conceptuels contradictoires et échappent donc à la définition des tropes proposée plus haut. Pour Prandi, de telles expressions ont un ressort inférentiel, procèdent par association d'idées habituelle (catachrèse) ou occasionnelle (métalepse), et ne mettent pas en relation contradictoire le contenu conceptuel des expressions engagées.

Le conflit conceptuel entre le nom et l'adjectif qui lui est apposé peut se résoudre soit par une réinterprétation métaphorique de l'adjectif (Un après-midi chenu), soit par une reconfiguration du rapport entre adjectif et nom. Dans ce cas, on a affaire à une synecdoque ou à une métonymie: le contenu conceptuel se dilate pour admettre une instance intermédiaire contiguë de l'adjectif et du nom. (Une mort blanche devient une mort provoquée par la neige, qui est blanche; $U n$ pas souriant devient le pas d'une personne souriante, ou bien le pas d'une personne dont l'arrivée provoque le sourire).

Dans les tropes qui impliquent une reconfiguration du rapport adjectival, on constate qu'il est difficile de départager les deux pôles de la contradiction entre cadre littéral et foyer figuratif: la mort et la blancheur, le pas comme le sourire, sont à prendre au premier degré. Ceci nous amène à nuancer les propos de Prandi sur le verbe, qui nous semble pouvoir supporter des tropes non métaphoriques lorsqu'il entre en contradiction avec son sujet ou son objet. Dans $I l$ a bu tous ses sous, le rapport métonymique entre dépenser et boire, complété par celui entre boisson et argent, interdit qu'on détermine, d'entre le verbe et son objet, celui qui est à prendre au sens figuré et celui qu'il faut entendre littéralement. 


\section{La mécanique des tropes est-elle formelle ou bien conceptuelle?}

A l'instar de Ricœur, Prandi oppose deux visions du fonctionnement tropologique (cf. schéma p. 56).

D'un côté, les tropologies substitutives, qui correspondent à la tradition classique et à la rhétorique d'expression française (Aristote, Fontanier, Nouvelle Rhétorique, Groupe Mu...) envisagent le trope comme substitution de forme. Les figures se résument au remplacement d'un signifiant par un autre.

Dans une telle conception, poussée à l'extrême par les auteurs d'après-guerre, la figure ne se conçoit que de manière négative, par oposition à un Degré Zéro non marqué. Elle se définit comme écart par rapport à une norme à la fois fonctionnelle (simple) et régulière (communément admise). Développant cette vision jusqu'à l'absurde, Prandi montre qu'elle entraîne à la fois le viol absolu du système linguistique par les figures et leur totale indépendance à son égard: les contemporains se coupent l'herbe sous le pied, puisque c'est la structure conceptuelle régulière qui, selon Prandi, permet de produire les conflits conceptuels où les tropes s'enracinent.

De l'autre côté, à l'opposé des tropologies substitutives, les métaphorologies interactives (Dumarsais, Ricœur, Black, Richards) conçoivent les tropes comme transfert de contenu ${ }^{1}$, comme transformation d'un sujet primaire, figuré, par un sujet secondaire, figuratif et exprimé littéralement (le concept de rossignol transforme le concept de fille en lui transférant certaines qualités). Selon Prandi, les interactionnistes définissent les tropes de manière positive, comme énoncés bénéficiant d'un supplément de structuration du contenu conceptuel ("surinvestissement de moyens formels").

1 Pour une autre définition des thèses interactionnistes, à partir de Black, Ricœur, Hausman et Johnson, cf. J. WAGONNER, "Interaction Theories, of Metaphor Psychological Perspective", in Metaphor and symbolic activity, 5 (2), p. 91-108. 


\section{FIGURE \\ SUBSTITUTIVE}

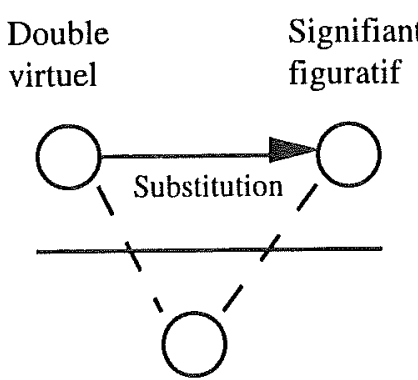

Contenu
TRANSFERT DE

CONTENU

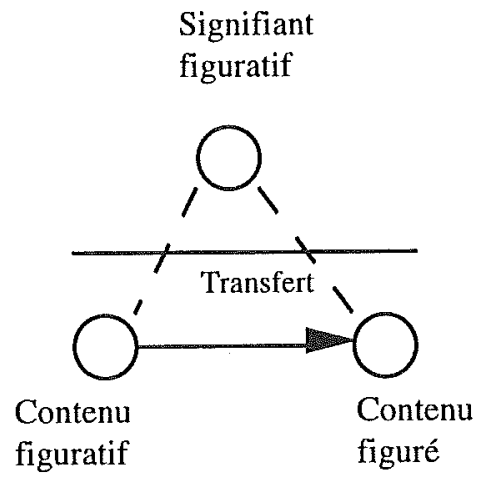

Pour les tropologies substitutives (à gauche), le signifiant figuratif est substitué, lors de l'encodage, à un signifiant littéral, de contenu identique. Celui-ci apparaît comme un "double virtuel" au récepteur qui décode la métaphore.

Pour les interactionnistes (à droite), une partie du contenu figuratif est transféré par la métaphore au sujet du discours figuré.

Figure 1

Valorisant la conception interactionniste, l'auteur souligne que le trope n'est figure (au sens que Fontanier réserve à ce terme) que dans le cas où l'expression tropique peut être remplacée par un signifiant littéral, c'est-à-dire par un double virtuel non tropique: une expression de même classe grammaticale, capable de remplir ses fonctions syntaxiques, conceptuellement équivalente, mais qui n'induit pas de contradiction syntaxique ou référentielle.

On pourra ainsi substituer crie à rugit dans L'homme rugit (tropefigure), alors que pour l'expression La lune rêve (trope non-substitutif), la langue n'offre pas de substitut verbal systématique pertinent.

Dans tous les cas le double virtuel n'intervient qu'après coup dans l'interprétation tropique: le récepteur s'en sert pour exprimer ce qu'il a compris, pas pour comprendre le trope. 


\begin{tabular}{|c|c|c|}
\hline $\begin{array}{l}\text { Figure (1) rhétorique } \\
\text { non tropique } \\
\text { "La claire pénombre" }\end{array}$ & \multicolumn{2}{|l|}{$\begin{array}{l}\text { Non-contradiction } \\
\text { ou bien } \\
\text { conflit sans solution }\end{array}$} \\
\hline \multirow{2}{*}{$\begin{array}{l}\text { Trope-Figure (1) } \\
\text { "L'homme rugit" } \\
\text { (Trope structurel) }\end{array}$} & \multirow{3}{*}{$\begin{array}{l}\text { TROPES: } \\
\text { Conflit } \\
\text { conceptuel } \\
\text { admettant une } \\
\text { solution par } \\
\text { transfert } \\
\text { synchronique }\end{array}$} & \\
\hline & & $\begin{array}{l}\text { FIGURES(1): } \\
\text { double virtuel } \\
\text { disponible en } \\
\text { langue }\end{array}$ \\
\hline $\begin{array}{l}\text { Trope non-substitutif (1) } \\
\text { "La lune rêve" } \\
\text { (Trope représentationnel) }\end{array}$ & & $\begin{array}{l}\text { NON-FIGURES } \\
\text { (1): } \\
\text { pas de double } \\
\text { virtuel disponible }\end{array}$ \\
\hline \multicolumn{2}{|l|}{-} & \\
\hline $\begin{array}{l}\text { Catachrèse } \\
\text { "l'aile du bâtiment" }\end{array}$ & $\begin{array}{l}\text { Transfert CONVENTION } \\
\text { diachronique, } \\
\text { non-contradiction }\end{array}$ & \\
\hline \multicolumn{3}{|c|}{$\begin{array}{l}\text { (1) Il nous semblerait préférable de continuer à employer le terme de figure pour } \\
\text { désigner l'ensemble des tournures canoniques de la rhétorique. En conséquence, } \\
\text { nous préférerions utiliser les expressions "trope structurel" et "trope } \\
\text { représentationnel" là où Prandi parle de "trope-figure" et de "trope non-substitutif", et } \\
\text { remplacer la distinction "figure/non-figure" par la distinction } \\
\text { "structure/représentation". }\end{array}$} \\
\hline
\end{tabular}

Figure 2

\section{Typologie interactionniste des tropes}

Comme nous l'avons vu plus haut, le fonctionnement des tropes repose sur une connexion, syntaxique ou référentielle, qui sous-tend une contradiction conceptuelle. La résolution de cette contradiction s'opère grâce à un transfert de contenu: le signifié littéral du foyer, sémantiquement incompatible avec le contenu conceptuel du cadre, est inhibé par la structure de l'énoncé. Un contenu figuratif est élaboré pour le remplacer. Ce contenu entretient avec le signifié originaire un rapport de ressemblance (métaphore), de contiguité (métonymie) ou d'inclusion (synecdoque).

Comme nous allons le voir à présent, le jeu conceptuel permettant la résolution de la tension peut prendre diverses formes. 
Réadaptant l'appareil conceptuel des interactionnistes', Prandi souligne la distinction entre d'une part le rapport foyer/cadre au plan de l'expression (signifiants figuratifs faisant figure sur le fond des signifiants littéraux), et d'autre part le rapport véhicule/teneur au plan du contenu ("sujet de discours secondaire", concept figuratif, décrivant un "sujet de discours primaire", concept figuré). Le tableau suivant montre l'application de cette double distinction à l'expression "Le rossignol que j'ai épousé".

\begin{tabular}{|lll|}
\hline EXPRESSION & FOYER: & CADRE: \\
& "Le rossignol" & "que j'ai épousé" \\
CONTENU & $\begin{array}{l}\text { VÉHICULE: } \\
\text { un rossignol }\end{array}$ & $\begin{array}{l}\text { TENEUR: } \\
\text { ma femme }\end{array}$ \\
& & \\
\hline
\end{tabular}

Figure 3

On peut ainsi distinguer quatre types de tropes:

Dans le trope in praesentia (Cette fille est un rossignol), le cadre correspond à la teneur et le foyer au véhicule, alors que dans le trope in absentia (J'ai épousé un rossignol), le cadre littéral ne désigne pas le sujet primaire, bien qu'il ressorte de son isotopie (épouser ne désigne pas la femme mais fait partie du même champ sémantique). Le référent est alors désigné pragmatiquement sans être formellement exprimé. Enfin, dans le trope cohérent (J'ai aperçu un rossignol) le cadre littéral est absent, la contradiction est référentielle.

1 Présentant rapidement les métaphorologies interactives de Black et Richards, Prandi se consacre principalement à en souligner les lacunes relatives à son propre point de vue (celui de la grammaire philosophique): chez les deux auteurs, absence de démarche proprement grammaticale; chez Black, limitation aux métaphores in absentia. 
In praesentia

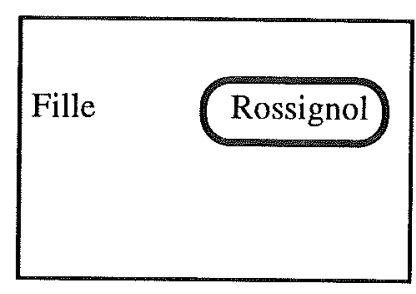

Trope cohérent

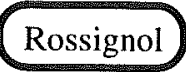

Rossignol

Femme

\section{In absentia}

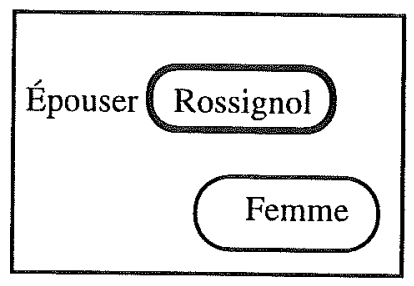




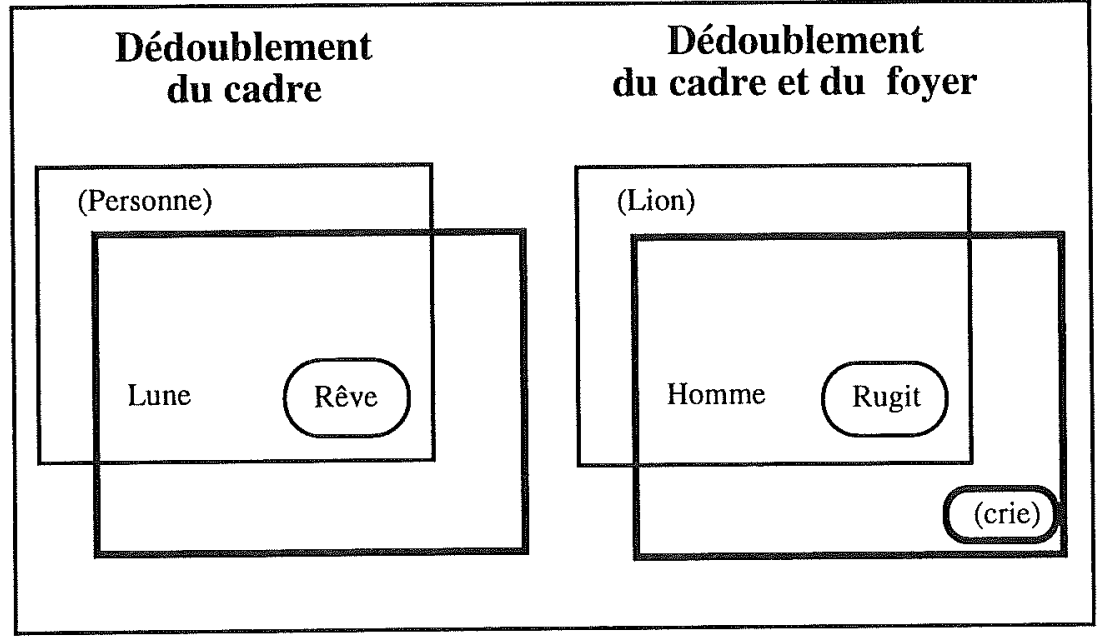

Figure 5

\section{Le fonctionnement inférentiel des tropes}

Parce qu'il y a toujours un certain degré d'indicalité ${ }^{1}$, un certain décalage à combler entre signifiant et référent, tout énoncé, même cohérent, entraîne un minimum d'inférence. Mais, dans le cas des énoncés contradictoires en général, et des tropes en particulier, l'inférence ne sert pas qu'à assurer l'indication, elle doit aussi servir à résoudre un conflit conceptuel: "La présence d'une interférence entre concepts hétérogènes exclut une interprétation littérale des tropes vivants: leur structure sémantique même impose et oriente ce travail interprétatif que les énoncés non tropiques se limitent à admettre" (p. 230).

Le seuil minimum d'inférence est donc plus élevé pour les énoncés contradictoires et tropiques. Dans le cas des tropes-figures, le supplément d'inférence peut se limiter à la substitution de la teneur en lieu et place du véhicule. Les tropes non-substitutifs, par contre, imposent un travail inférentiel plus important encore, offrent plus de résistance à l'interprétation.

En fonction des relations préexistantes entre teneur et véhicule, Prandi différencie deux types d'analogies: d'une part, l'analogie définie, basée sur un vecteur, une relation préexistante entre concepts

1 Pour un examen approfondi de la notion d'indicalité, cf. infra, point 8. 
figurant et figuré, d'autre part, l'analogie projective, qui crée de nouvelles relations (cette distinction ne recouvre pas celle qui oppose les conceptions substitutives et interactives de la métaphore). “... Saisir le degré d'incidence de l'analogie projective revient à déterminer dans quelle mesure la reconfiguration de la teneur se réduit à une pure et simple reproposition de propriétés focalisées dans le véhicule préalablement et indépendamment de la métaphore donnée, et dans quelle mesure la recherche d'analogies nous amène à découvrir, ou du moins à focaliser, des propriétés non banales du véhicule projetables sur la teneur" (p. 241). L'analogie projective comprend les tropes non-substitutifs qui transcendent les stéréotypes (Ta chevelure est une rivière tiède) et les catachrèses d'auteur (cf. infra, point 7 , typologie des métaphores).

Parmi les analogies définies, Prandi oppose analogie structurale et analogie conceptuelle. L'analogie définie structurale, basée sur les relations systématiques entre lexèmes, correspond à la catégorie du trope-figure ( $L$ 'homme rugit). L'analogie définie conceptuelle se base sur le "système de lieux communs", associés aux concepts, c'est-àdire sur les stéréotypes de l'ontologie. Ainsi Ce type est un perroquet évoque directement le fait qu'il répète les paroles d'autrui parce que la répétition est une des propriétés prototypiques du perroquet.

A ces trois types de tropes correspondent trois types de lectures. Prandi distingue ainsi trois niveaux croissants de travail inférentiel: simple identification de la teneur, activation des lieux communs de l'ontologie du véhicule avec transfert à la teneur, développement original de l'interaction conceptuelle entre teneur et véhicule.

Toutefois, un type de trope donné n'entraîne pas forcément un seul type de lecture, il peut donner lieu à des développements aux niveaux supérieurs: "Une analogie définie a de bonnes probabilités de s'imposer (...), grâce à son inertie. Toutefois, sa pertinentisation (...) étant à son tour subordonnée à l'examen de l'inférence, elle n'en demeure pas moins révoquable, et remplaçable par la construction d'analogies projectives qui la dépassent" (p. 242).

Par exemple L'homme rugit est trope-figure (on peut substituer crie à rugit ), mais comporte un aspect projectif, suggère que le cri présente certaines propriétés inhérentes typiques du rugissement, ou que l'homme a le courage, la crinière, la force ou encore la violence 
du lion. Une telle inférence projective implique des représentations auditives, visuelles et comportementales... en un mot, ontologiques ${ }^{1}$.

\section{Typologie des métaphores}

Nous sommes à même à présent de distinguer avec Prandi quatre catégories de tropes:

- le trope-figure structural (l'homme rugit);

- le trope non-substitutif conceptuel (C'est un perroquet);

- le trope non-substitutif projectif (Ta chevelure est une rivière tiède);

- la catachrèse lexicale (L'aile du bâtiment).

Prandi conçoit trois catégories supplémentaires, réservées à la métaphore, destinées à l'expression de vérités empiriques ou nouméniques.

- La catachrèse d'auteur (Une révolution scientifique) est une création qui permet de créer un nouveau concept en projetant dans l'isotopie de la teneur les structures imaginaires propres à un véhicule d'un autre ordre. Elle se distingue des catachrèses en général parce qu'elle ne provient pas d'une sédimentation progressive dans le lexique (diachronie), mais d'une invention ponctuelle. Elle a cependant le caractère transparent de la catachrèse lexicale, puisqu'elle permet une résolution totale et immédiate du conflit conceptuel. La

1 Prandi nous permet ainsi de proposer une solution originale au débat portant sur la structure du processus cognitif sous-jacent à la métaphore, à propos duquel s'affrontent les psychologues expérimentaux. La théorie du contraste de saillance d'Ortony (A. ORTONY, "Beyond literal Similarity", in Psychological Review, 86, 1979 , p. 161-180) sera plus facilement confirmée par des analogies conceptuelles, alors qu'un matériel expérimental induisant des analogies structurales favorisera le modèle par catégorisation de Glucksberg (S. GLUCKSBERG and B. KEYZAR, "Understanding litteral Comparisons: beyond Similarity", in Psychological Review, 97,1990 , p. 3-18). Quant à la question de la préexistance sémantique (A. ORTONY, op. cit.; A. TVERSKY, "Features of Similarity", in Psychological Review, 85, 1977, p. 327-352) ou de l'émergence (M. CAMAC and S. GLUCKSBERG, "Metaphors do not use associations between concepts, they are used to create them", in Journal of Psycholinguistic Research, vol. 13, No 6, 1984, p. 443-455; J. WAGONNER, op. cit.) du contenu de la métaphore dans la teneur et le véhicule, elle recevra une réponse différente selon qu'on observe des analogies projectives suscitant l'interaction conceptuelle ou des analogies transférant des propriétés via des vecteurs prédéfinis. Il semble donc que les exclusives posées par les psychologues doivent plutôt être considérées comme des alternatives. 
catachrèse d'auteur n'est pas substitutive: on ne peut la remplacer par un équivalent lexicalisé, le concept qu'elle évoque n'existe pas en dehors de l'expression catachrétique.

- La catachrèse institutive (Code génétique) est une catachrèse d'auteur réservée à une discipline scientifique, dont elle assoit les bases conceptuelles.

- Les métaphores illustratives (Les archives de la mémoire, Le courant électrique) sont elles aussi transparentes, mais désignent de manière pédagogique des concepts préexistant à la métaphore. Ce n'est pas le transfert de qualités nouvelles qui est ici valorisé, mais les traits communs préexistants entre sujets de discours primaire et secondaire. Il s'agit en fait d'une métaphore conceptuelle à vocation explicative.

Selon nous, il faut rajouter aux trois types précités les variantes d'auteur, créations langagières réexploitant des relations systématiques que Lakoff et Johnson désignent sous le terme d'invariants ${ }^{1}$ : es invariants regroupent de nombreuses projections de même type, reliant deux mêmes réalités (p. ex., l'amour et le feu) et déjà exprimées par un ensemble de catachrèses lexicalisées et d'expressions stéréotypées (Le feu de la passion, Déclarer sa flamme, Brûler de désir, Un regard de braise). Toute création exploitant le même invariant (L'étincelle de la rencontre) peut être considérée comme une variante d'auteur, cas particulier de la catachrèse d'auteur.

Aux huit catégories précitées Prandi superpose une distinction, réservée elle aussi à la métaphore, et établie en fonction du type de contenu transféré. Une analogie ponctuelle, comme Cette fille est le rossignol de l'école, établit une ressemblance qualitative entre concepts. Une analogie proportionnelle (Cette fille est le bouc émissaire de l'école), établit une ressemblance de rapports, transfère une relation depuis une paire de concepts (le bouc émissaire et la population juive) jusqu'à l'autre (la fille et la population de l'école).

1 La dimension cognitive des tropes est abordée par Prandi sans références à la psycholinguistique ou à la sémantique cognitive. Lorsque l'auteur aborde l'hypothèse d'invariance (p. 239-240), il ne fait pas référence à George LAKOFF et Mark Johnson, Les métaphores dans la vie quotidienne, Paris, Éd. de Minuit, 1985, ni aux ouvrages qui en découlent. 


\section{Le rôle du contexte dans l'interprétation}

Le conflit conceptuel qui sous-tend un trope peut être structural (L'homme rugit), ontologique (La lune rêve), ou purement contextuel (Cet homme est un charcutier, en parlant d'un chirurgien).

Cependant, la résolution de ce conflit se fait, selon Prandi, sur une base toujours contextuelle (pour notre part, les régularités propres aux représentations figurées permettent d'envisager un niveau autonome de résolution à base cognitive, en fonction de données ontologiques).

Comme le montre Prandi, la distinction entre énoncé cohérent et contradictoire ne peut se fonder sur des critères purement formels, certaines phrases ne sont qu'occasionellement contradictoires: leur incohérence n'est déclenchée que par inférence. C'est le cas des connexions référentielles et des énoncés structurellement ambigus nécessitant une interprétation interne basée sur des facteurs pragmatiques externes.

\section{L'indicalité de la communication langagière: une thèse}

A l'arbitraire de la signification, Prandi propose d'ajouter comme principe général une indicalité de la communication langagière:

"Aucune relation structurellement fondée ne relie le contenu linguistique de l'énoncé aux développements inférentiels différents qu'il autorise dans les contextes différents. En nous inspirant de Peirce et de Bühler, nous suggérons que la relation entre le contenu des expressions et les messages échangés est une relation d'indication: les signifiés linguistiques, reliés à leurs signifiants par une relation structurale relevant de l'ordre symbolique ${ }^{1}$, circulent dans la communication comme indices de messages contingents" (p. 144).

"L'hypothèse d'un lien d'indication entre signifié et message garantit (...) la continuité fonctionnelle entre signification et communication, sans compromettre leur autonomie réciproque, qui est au contraire présupposée. (...). Le processus de communication, pour sa part, acquiert la dignité d'un domaine autonome, étranger aux struc-

1 Le terme de symbole est ici à entendre au sens Peircéen, digital et arbitraire, et non pas au sens Saussurien, analogique et motivé. 
tures linguistiques spécifiques, ce qui justifie son aptitude à conférer aux signifiés linguistiques leur valeur propositionnelle d'indices" (p. 152). Confronté à la connexion contradictoire, l'interprète prend le risque d'attribuer un sens à l'énoncé en recherchant, dans le contexte d'interprétation, un référent relié au signifié du foyer tropique par une relation d'indication.

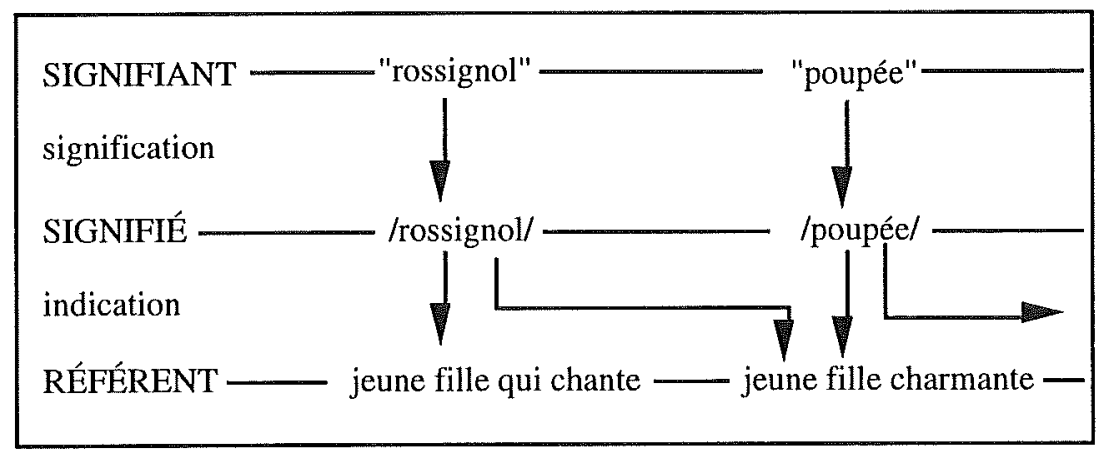

Figure 6

Reprenant les thèses de Sperber \& Wilson, Prandi décrit l'interprétation des énoncés comme un travail actif de sélection, fondé sur une évaluation ad hoc des circonstances de l'acte de communication, parmi les éléments du champ d'énonciation. Le récepteur est donc engagé de manière responsable dans la construction d'un environnement cognitif ${ }^{1}$ pertinent pour l'interprétation.

\section{Conséquences éthiques de l'indicalité de la communication langagière}

Pour Prandi, dans l'interaction face-à-face, la communication vise avant tout l'adéquation entre intentions communicatives et interprétations. Dans l'interaction, le travail inférentiel constitue une manière d'atteindre cette adéquation, alors que dans l'écrit la production d'inférences peut constituer une fin en soi.

1 La notion d'environnement cognitif est empruntée à Dan SPERBER et Deirdre WILSON, La pertinence, communication et cognition, Paris, Éd. de Minuit, 1989. 
Fondée sur un paradoxe, la communication suppose en même temps l'altérité radicale entre individus et la réversibilité des rôles discursifs. Dans l'interaction communicative, l'équilibre délicat entre distance et empathie joue un rôle clé: l'excès de fusion mimétique conduit à l'irrespect, mais son absence entrâne elle aussi la violence.

Les notions de sens littéral et d'interprétation authentique sont d'ordre pragmatique et non formel. La transparence du signe, que les locuteurs considèrent comme un fait de langue, est une illusion. Mais cette "illusion nécessaire" permet de fonder la règle éthique bien réelle de transparence de la communication, qui vise l'adéquation, toujours à construire, entre intention et interprétation.

On retrouve ici des préoccupations proches de celles de Sperber et Wilson, pour qui l'acte de langage est un risque calculé, et son interprétation une stratégie inférentielle qui prend en compte ce risque, inhérent à la communication.

Actualisées dans les énoncés, les expressions langagières possèdent à la fois un contenu conceptuel virtuel et une référence actuelle. Lorsque la dénotation virtuelle contredit la désignation actuelle, ou lorsqu'elle est insuffisante à confirmer la référence, on parle de désignateur obscur (tropes, ironie, allusion, etc...). Les tropes ne constituent qu'un cas particulier de désignateurs obscurs, qui peuvent, le cas échéant, organiser des dénotations contradictoires (via une connexion syntaxique) et donc être inappropriés à la référence avant même d'être contextualisés.

Selon la rhétorique classique, on produit un trope par substitution d'un désignateur obscur à un désignateur transparent préexistant. Selon Prandi, la substitution concerne l'interprétation et non la production des tropes. Elle n'est qu'une renomination, a posteriori, et à l'aide d'un désignateur transparent, du référent du trope. Alors qu'il a déjà compris le trope, l'interprète reformule le référent en termes explicites pour mieux s'en assurer la maîtrise.

En tant qu'elle vise à identifier un référent, une expression peut donc être transparente ou obscure. En tant qu'elle le qualifie, l'expression peut être neutre ou conceptuellement engagée. Dans le premier cas, elle se réduit à une simple désignation. Dans l'autre, elle possède un contenu prédicatif dense qui implique un travail d'inférence au-delà de la désignation. Selon Prandi, les tropes vivants sont des expressions à la fois obscures et conceptuellement engagées. Elles ne permettent que difficilement l'identification mais recèlent un grand potentiel de qualification. 
Ceci nous suggère un parallèle avec Sperber et Wilson, pour qui la pertinence d'un message est proportionnelle à son informativité et inversément proportionnelle à son opacité. Dans ce cadre, les tropes paraissent concéder une augmentation d'opacité afin d'augmenter l'informativité.

\section{Critique: l'imagination comme complément nécessaire de l'indication}

Comme on le voit, Prandi propose, à l'occasion d'une étude sur les tropes, une théorie complète et cohérente de la communication langagière. Nous espérons avoir ci-dessus réussi à en formuler une synthèse à la fois claire et aussi fỉèle que possible.

Nous avons cependant une critique à formuler. Dans l'interlude de l'ouvrage, l'auteur, désireuse d'assurer la transition vers la section 2 à portée herméneutique, oublie d'intégrer dans son discours les propos fort pertinents développés dans la section 1 à propos de l'ontologie. L'interlude n'intègre donc que les aspects structuraux et pragmatiques de la communication, l'auteur n'y adjoint pas ses considérations sur l'imagination.

$\mathrm{Si}$ des déictiques purs comme les pronoms démonstratifs sont dépourvus de contenu conceptuel perceptif, les verbes, adjectifs et noms nécessaires à la mise en place des tropes ne fonctionnent généralement que grâce aux modèles mentaux intuitifs qu'ils mobilisent. Ainsi, selon nous, les concepts ne sont pas intelligibles sur base de leur seul signifié structural, celui-ci ne constitue qu'un relais vers les modèles mentaux qui sont au centre du contenu conceptuel.

Une métaphore comme Le naufrage du soleil impose, de manière invariable et dans la plupart des contextes d'énonciation, la superposition de deux Gestalt visuelles analogues, celle du soleil disparaissant derrière l'horizon marin et celle du navire sombrant dans la haute mer.

Une telle association repose sur cette dimension extralinguistique des concepts, que l'auteur lui-même aborde en termes d'ontologie. Il serait abusif de dire que le naufrage entretient avec le couchant une relation d'indication. Il y a bien, en effet, une indication stéréotypée entre le signifiant-foyer naufrage et une certaine Gestalt visuelle, sollicitée pour l'occasion. Mais la relation la plus essentielle dans ce trope est une relation intense d'analogie figurative entre cette même 
Gestalt visuelle et une autre Gestalt, indiquée, de manière tout aussi stéréotypée, par le signifiant-cadre soleil.

Plutôt que de considérer l'indicalité comme le paradigme canonique de la communication langagière, il nous semble préférable de concevoir, d'une part, la désignation pragmatique pure (déictique ou anaphorique), et, d'autre part, la figuration ontologique pure (poésie) comme deux pôles entre lesquels s'étend un continuum qui admet diverses nuances.

Pour les cas les plus généraux, nous considérons que les données ontologiques imaginaires évoquées par les lexèmes s'unissent à celles que le contexte manifeste, et que les deux mécanismes fonctionnent de manière complémentaire et comblent leurs lacunes réciproques dans le but de créer un modèle mental cohérent.

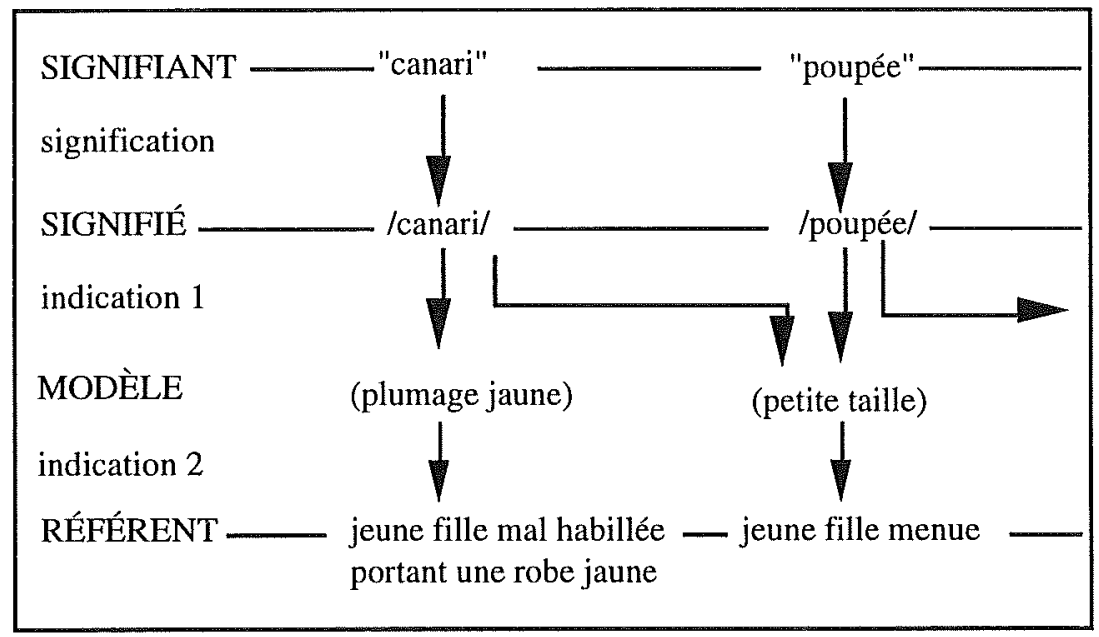

Figure 7

Si modèle et référent sont aussi importants l'un que l'autre pour l'élaboration du contenu des énoncés, nous pensons cependant que, dans le cas particulier des tropes créateurs, le rôle des modèles mentaux est toujours primordial. Même dans le cas des connexions référentielles, les représentations du véhicule, liées au foyer figuratif, recèlent pour nous l'essentiel du contenu conceptuel transféré au référent. Fût-elle fortement dépendante du contexte, une expression tropique créative fait pour nous toujours appel à l'imagination.

A cette affirmation, on pourrait objecter que la catachrèse et le trope structural se passent des représentations. Mais c'est justement 
parce qu'ils s'en passent qu'ils ne peuvent selon nous être considérés comme tropes au sens que Prandi réserve à ce terme (qui implique l'existence d'une contradiction amenant le récepteur à découvrir une teneur derrière un véhicule).

Une question à résoudre dans ce cadre est celle de l'extension qu'il faut donner au concept d'imagination. Doit-on y inclure uniquement des schémas perceptifs et proprioceptifs? Ou bien peut-on y ajouter les scripts qui sont à la base de nos actions, les scénarios qui forgent notre compétence narrative et les sensations, éventuellement synesthésiques, qui constituent la base de notre intuition? En ce qui nous concerne, nous choisissons d'inclure sous le terme d'imagination toutes ces formes de contenu qui résiste à la factorisation commutative des sémantiques structuralistes.

Notre capacité à représenter le monde sans recourir au langage est tout naturellement à la source du renouvellement de la langue. De son vivant, le trope, avant que les outrages du temps ne le cristallisent en catachrèse, manifeste la nécessité d'une intrusion de l'irrationnel dans l'ordre structural. Sans cette ouverture à l'intuition, toutes les langues du monde se révéleraient muettes, à jamais incapables de dire l'indicible. 